\title{
Anomalous behavior of ultra-relativistic particles - a phenomenon that can confirm the discreteness of time
}

\author{
Alexander Zaslavsky ${ }^{1,2}$ \\ ${ }^{1}$ National Mining University, Department of Information Technology, Dnipropetrovs'k, Ukraine \\ ${ }^{2}$ Web-Research Institute of the Nature of Time at Moscow State University, Moscow, Russia
}

\section{Email address:}

am-47@mail.ru, amz.nmu@gmail.com

\section{To cite this article:}

Alexander Zaslavsky. Anomalous Behavior of Ultra-Relativistic Particles - a Phenomenon That Can Confirm the Discreteness of Time. American Journal of Modern Physics. Special Issue: Physics of Time: Theory and Experiment. Vol. 4, No. 2-1, 2015, pp. 34-40. doi: 10.11648 j.ajmp.s.2015040201.16

\begin{abstract}
The paper analyses physical phenomenon which can give evidence of discreteness of time. The phenomenon is substitution of wave function of ultrarelativistic particle in terms of certain (hyper-ultrarelativistic) values of its space motion velocity. Discreteness of time is necessary criterion for such a substitution. The paper has developed mathematical description for particle energy (frequency) anomalous dependence on its velocity in the context of frequency substitution resulting from discreteness of time. Moreover, there have been obtained estimations of maximum energy of a particle of the given mass, maximum particle mass as well as a value of its own time quantum.
\end{abstract}

Keywords: State, Stream of Events, Time Quantum, Wave Function, Frequency Substitution, Folds, Energy

\section{Introduction}

Practically each recognized theory of classic and modern physics considers time as both infinitely divisible (dense) and continuous. However, everyone understands that the assumption can be reconsidered. The matter is that it has not been proved being supposed as obvious. The problem of fundamental length and fundamental time existence has become quite popular recently [1-5]. However, only experimentally confirmed physical effect being direct result of discreteness of time is a serious reason for such revision of basics of physics. The paper is devoted to the analysis of possibility to demonstrate physical essence of this effect. Its idea is to study from the point of view of general physical applications the phenomenon of frequency substitution known in applied problems of theory of time series [6]. This phenomenon is seen in anomalous changes in a signal frequency spectrum restored according to the selected values obtained in discrete time moments. Reduction of sampling frequency as for signal frequency being lower than critical value known as Nyquist frequency is its condition of origin [7] General physical context means substitution of a particle wave function in terms of its movement velocity change being responsible for frequency of wave function as well as its selected values repetition rate in discrete time. If this physical effect is experimentally confirmed then reconsideration of continuous time concept will be complete. If it is possible to obtain achieve firm evidences of its impossibility then time continuity will be proved. It should be noted that for this case the notion of "negative result" cannot be used. Both alternative results are similarly valuable not only for physics but also for science in general taking into account fundamental values of Time for natural science as a whole.

\section{Sampling Rate of Time in Inertial Frames of Reference}

Special relativity theory uses a notion of own time of point object (particle). That means clock time being fixed in frames of reference connected with the object. Geometrical interpretation of the theory identifies own time with the interval of the object world line within spacetime. However, if clock (as an event meter) is taken into account then world line should be considered as discrete sequence of points - events, which can be interpolated with a line if only it is correlated with two events following each other, the least possible interval - own time quantum. As for space it is supposed as continuous. Considering a particle as a sequence of events in time we exclude possibility to find two or more its different states within one and the same time moment which cannot 
exclude possibility to find a set of its different (at different times) states either in one spatial point or in its infinitely small neighborhood. It means that time interval between two events of one particle whatever small it is cannot be equal to zero when a segment connecting two events in space can be infinitely small up to zero length.

Let frame of reference of observer is connected with a point particle located in the initial part of space coordinates. In this case ranked set (stream) of events $\left(x_{0}, y_{0}, z_{0}, t_{0}\right),\left(x_{0}, y_{0}, z_{0}, t_{1}\right),\left(x_{0}, y_{0}, z_{0}, t_{2}\right) \ldots \quad$ is called a particle. Discrete time is the object of our study. Hence, time interval $t$ between two given events is measured by the number of events included between them

$$
t=\tau \Delta t_{0}
$$

where $\tau \in(1,2, \ldots)$ is the number of events separating those events between which time $t$ is measured; $\Delta t_{0}$ is a constant specifying metric of time axis - time quantum. The constant value is supposed to be arbitrarily small but not equal to zero.

Substituting minimum $\tau=1$ value for (1) we obtain that time quantum is the shortest interval of observer's own time. It means that the duration of time quantum cannot be principally measured within its own time. Let assume that there is the second frame of reference connected with the second observer. Similarly to case one own discrete time of the second observer $t^{\prime}$ is measured by the number of elementary events $\tau^{\prime}$ of a particle being fixed relative to the observer identified with time moments

$$
t^{\prime}=\tau^{\prime} \Delta t_{0}^{\prime}
$$

Therefore $\Delta t_{0}^{\prime}$ constant is also cannot be measured at the own time of the second observer. That is time quantum of the first observer can be principally measured at the own time of the second observer but not in terms of own time. For example, using my own clock I can measure average length of elementary interval (the duration of the second) in a clock of my interlocutor. She/he can do the same as for my clock using own one; however, it is impossible for me to measure the duration of the second in my clock using time being shown by it.

Hence own time quantum with accuracy of measurement units is universal constant $\left(\Delta t_{0}^{\prime}=\Delta t_{0}=\right.$ const $)$ just as a result of immeasurability within own time.

If a particle is represented by a regular stream in which events are separated from each other by similar time intervals equal to $\Delta t_{0}$, then the particle event stream is a stream of its own time. This very particle being considered by outer observer within another reference system is a certain stream of random events being observed from the outside. In a general case, events within the stream can be separated from each other by different random time intervals. While generalizing we can say that the thing being own time for a particle is a stream of observed object events for the outer observer; generally it is irregular.

Let us agree that values belonging to the object being observed are identified as $k$ and values belonging to an observer have no indices. Mark as $\tau_{k}(t, \Delta t)$ a random quantity of events of an object being observed within $(t, t+\Delta t)$ time interval measured by an observer. From now on it is assumed that an observer objectively reflected the reality registers each state of an object being observed. Distribution of random variables $\tau_{k}(t, \Delta t)$ is

$$
\tau_{k}(t, \Delta t):\left|\frac{0}{p_{0}^{k}(t, \Delta t)} \| \frac{1}{p_{1}^{k}(t, \Delta t)}\right||\ldots|,
$$

where in upper columns (above the line) possible values of quantities of events ...2, $3, \ldots$, are located and below the line probability values $p_{2}^{k}(t, \Delta t), p_{3}^{k}(t, \Delta t), \ldots$ corresponding to them are located. For any pair $(t, \Delta t)$ normalization requirement is done

$$
p_{0}^{k}(t, \Delta t)+p_{1}^{k}(t, \Delta t)+p_{2}^{k}(t, \Delta t)+\ldots=1 .
$$

Mathematical expectation of a random variable $\tau_{k}(t, \Delta t)$ is equal to the sum

$$
M_{k}\left(\tau_{k}\right)=0 \cdot p_{0}^{k}(t, \Delta t)+1 \cdot p_{1}^{k}(t, \Delta t)+2 \cdot p_{2}^{k}(t, \Delta t)+\ldots .
$$

Considering a particle as an event stream, assume it as an ordinary one. It means that probability of two or more events of one and the same particle origin within a small time interval $\left(\Delta t \rightarrow \Delta t_{0}\right)$ tends to zero (events follow each other strictly). Hence, in the limit, mathematical expectation $M_{k}\left(\tau_{k}\right)$ tends to the value

$$
\mu_{k}(t)=\lim _{t+\Delta t \rightarrow t} M_{k}\left(\tau_{k}\right)=p_{1}^{k}(t, \Delta t)
$$

Express increment of own time of observation object through the increment of observer's time putting into (1) the mathematical expectation $\mu_{k}(t)$ of the number of events of observation object within small time interval

$$
\Delta t_{k}=\mu_{k}(t) \Delta t_{0}
$$

Having divided both parts of the equality by $\Delta t$, find the relation value of own times of observation object and an observer $\Delta t_{k} / \Delta t$

$$
\frac{\Delta t_{k}}{\Delta t}=\frac{\mu_{k}(t)}{\Delta t} \Delta t_{0}
$$

In a theory of random processes, value $\sigma_{k}(t)=\mu_{k}(t) / \Delta t$ is called average density (intensity) of event stream. It is average event frequency within $(t, t+\Delta t)$ segment. Hence, expression (8) can be represented as 


$$
\frac{\Delta t_{k}}{\Delta t}=\sigma_{k}(t) \Delta t_{0}
$$

Estimate a relation value of own times of observation object and an observer. As $\Delta t_{0}$ is the least possible interval of own time ( $\left.\Delta t_{0} \leq \Delta t\right)$, the inequality follows from (8)

$$
\frac{\Delta t_{k}}{\Delta t} \leq \mu_{k}(t)
$$

Taking into account (6), we obtain

$$
\frac{\Delta t_{k}}{\Delta t} \leq p_{1}^{k}(t, \Delta t) \leq 1
$$

The given inequality means that velocity of own discrete time of observation object is not more than that one of an observer's. If within each time interval $\Delta t_{0}$ an observer finds instantaneous state of an observation object with a probability of 1 , it means that their times flow similarly. If $p_{1}^{k}(t, \Delta t)<1$, then own time of observed object flows slower than observer's time.

Fig. 1 shows a stream of particle events moving within space.

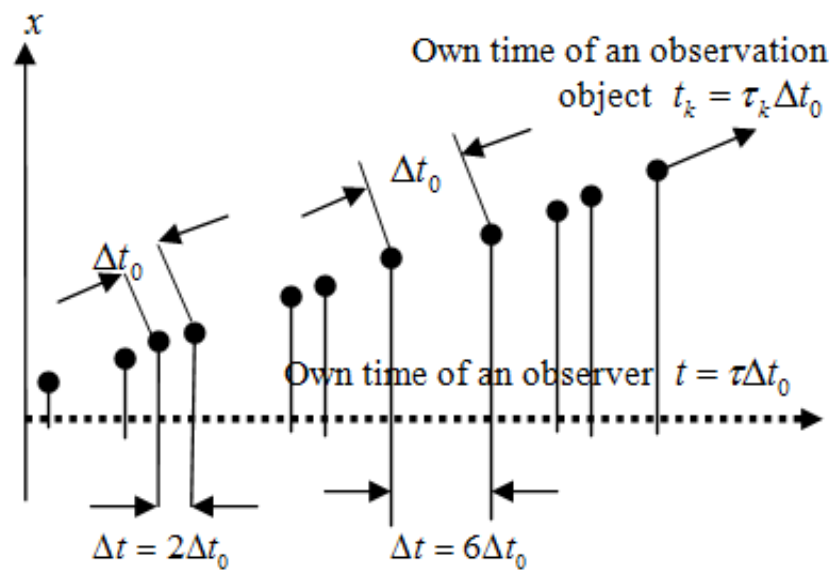

Fig. 1. A particle as a flow of discrete events.

If frame of reference connected with a particle performs continuous move in continuous space relatively to the observer's reference system with $V_{k}$ speed, then according to the known rules of special theory of relativity ratio of own time of a particle to observer's time is

$$
\frac{\Delta t_{k}}{\Delta t}=\sqrt{1-\frac{V_{k}^{2}}{c^{2}}}
$$

Strictly speaking studying a particle within discrete time one cannot consider its motion as continuous one. However, in terms of high density of events their discrete sequence can be approximated by a world line of a particle moving with $V_{k}$ speed within an observer's reference system. Comparing expressions (12) and (9), we can develop a formula connecting density of a particle event stream (discretization frequency) and its speed of motion within inertial frame of reference

$$
\sigma_{k}=\frac{1}{\Delta t_{0}} \sqrt{1-\frac{V_{k}^{2}}{c^{2}}} .
$$

\section{On Anomalous Dependence within Discrete Time and Continuous Space of Particle Energy upon Its Speed}

Wave function of a free particle within continuous spacetime is

$$
\psi(\mathbf{r}, t)=A \exp \{j(\omega t-\mathbf{k r})\}=\psi_{0}(\mathbf{r}) \exp (j \omega t),
$$

where $\omega=2 \pi \nu$ is wave frequency, $\mathbf{r}$ is a radius-vector of any spatial point, $t$ is time, $\mathbf{k}$ is a wave vector, $\psi_{0}(\mathbf{r})$ is time independent share of a complete wave function. Both frequency and wave vector are connected with particle energy and impulse with the help of known $E=\hbar \omega, \mathbf{p}=\hbar \mathbf{k}$ ratios of de Broglie.

If space is assumed as continuous and time is assumed as discrete then wave function should be represented in the form of selected (generalized) function

$$
\psi(\mathbf{r}, \tau)=\int_{-\infty}^{\infty} \psi_{0}(\mathbf{r}) \exp (j \omega t) \delta\left(\tau \Delta t_{0}-t\right) d t
$$

where $\delta\left(\tau \Delta t_{0}-t\right)$ is a delta function.

Thus, sample values of wave function are a sequence of functions of continuous space coordinates corresponding to discrete $\tau \Delta t_{0}$ time moments.

The main problem arising due to time discreteness is in the fact to what extent the sample values of wave function represent the initial wave function. According to the known sampling theorem [6], $\psi(\mathbf{r}, t)$ function can be restored uniquely on the basis of the sequence of $\psi(\mathbf{r}, \tau)$ functions if $\sigma_{k}$ is such discretization frequency that

$$
\frac{\omega}{2 \pi} \leq F=\frac{\sigma_{k}}{2}
$$

where $F$ is Nyquist frequency.

Putting $\sigma_{k}$ value from (13) we obtain following inequality

$$
\frac{1}{\Delta t_{0}} \sqrt{1-\frac{V^{2}}{c^{2}}} \geq \frac{\omega}{\pi}
$$

Hence

$$
\frac{|\mathbf{V}|}{c} \leq \sqrt{1-\frac{\omega^{2}}{\pi^{2}} \Delta t_{0}^{2}}
$$

The result can be interpreted as following. Until the module of particle speed is less than 


$$
c_{F}=c \sqrt{1-\frac{\omega^{2}}{\pi^{2}} \Delta t_{0}^{2}}
$$

wave function characterized by $\omega$ frequency can be uniquely restored according to discrete series of its instantaneous values following each other with intensity

$$
\sigma_{k}=\frac{1}{\Delta t_{0}} \sqrt{1-\frac{V^{2}}{c^{2}}}
$$

Let us see what happens if speed of a particle is more than $c_{F}$. To do that, express discrete time of a particle $t$ observation through average frequency of its own events.

Observation time of discrete sequence of events following each other with $\sigma_{k}$ frequency is proportional to the number of $\tau$ events and inversely proportional to $\sigma_{k}$ frequency at which they origin.

$$
t=\frac{\tau}{\sigma_{k}}, \tau=0,1,2, \ldots
$$

Putting this expression $t$ into wave function we obtain

$$
\psi=\psi_{0}(\mathbf{r}) \exp \left(j \frac{\omega}{\sigma_{k}} \tau\right) .
$$

Show $\omega / \sigma_{k}$ ratio in the form of integral part $z$ and fractional part $q$ of $\pi$ where $z$ number is multiplicity of Nyquist frequency excess $(z=0$ means that Nyquist frequency is not exceeded and $z=1,2, \ldots$ means that in terms of given time discreteness Nyquist frequency could be exceeded by more than $1,2, \ldots$ etc. times respectively)

$$
\frac{\omega}{\sigma_{k}}=z \pi+q \pi
$$

In this context wave function transforms as follows

$$
\psi=\psi_{0}(\mathbf{r}) \exp (j z \pi \tau) \exp (j q \pi \tau)
$$

Hence two possibilities arise depending upon number $z$ evenness and oddness.

1) If $z$ is even then $\exp (j z \pi \tau)=1$ for each $\tau$ corresponding to $\psi=\psi_{0}(\mathbf{r}) \exp (j q \pi \tau)$ wave function. Using (17) find angle $q \pi \tau$ for this case taking into account that

$$
\begin{gathered}
\tau / \sigma_{k}=t \\
q \pi \tau=\left(\omega-z \pi \sigma_{k}\right) t=\omega^{\prime \prime} t, \quad \mathrm{z}=0,2,4, \ldots .
\end{gathered}
$$

Hence it is clear that $\omega=2 \pi v$ frequency is substituted for the frequency

$$
\omega_{z}^{\prime \prime}=\omega-\pi \sigma_{k} z=\omega-\frac{\pi}{\Delta t_{0}} z \sqrt{1-\frac{V^{2}}{c^{2}}}, \quad \mathrm{z}=0,2,4, \ldots
$$

2) If $z$ is odd, then $\exp (j z \pi \tau)=(-1)^{\tau}$ for each $\tau$ If so, take into account following: $(-1)^{\tau}=\cos (\pi \tau), \quad \sin (\pi \tau) \equiv 0$.

Using Euler correlation, represent complex number $\exp (j z \pi \tau) \exp (j q \pi \tau)$ trigonometrically having added it with known zero addends

$$
\begin{aligned}
& \exp (j z \pi \tau) \exp (j q \pi \tau)=\cos (\pi \tau) \cos (\pi q \tau)+ \\
& +j \cos (\pi \tau) \sin (\pi q \tau)+\sin (\pi \tau) \sin (\pi q \tau)- \\
& -j \sin (\pi \tau) \cos (\pi q \tau)=\cos (\pi \tau(1-q))-j \sin (\pi \tau(1-q)) .
\end{aligned}
$$

Thus, we have $\exp (j z \pi \tau) \exp (j q \pi \tau)=\exp (j \pi \tau(1-q))$ for odd $z$.

Represent angle $\pi \tau(1-q)$ as follows

$$
\pi \tau(1-q)=\left(\pi \sigma_{k}-\left(\omega-z \pi \sigma_{k}\right)\right) \frac{\tau}{\sigma_{k}}, \mathrm{z}=1,3, \ldots
$$

In this case wave frequency is substituted for the frequency

$$
\omega_{z}^{\prime}=\pi \sigma_{k}-\omega_{z}^{\prime \prime}=\frac{\pi}{\Delta t_{0}}(z+1) \sqrt{1-\frac{V^{2}}{c^{2}}}-\omega, \quad \mathrm{z}=1,3, \ldots .
$$

If $z=1$, then we obtain

$$
\omega_{1}^{\prime}=\frac{2 \pi}{\Delta t_{0}} \sqrt{1-\frac{V^{2}}{c^{2}}}-\omega
$$

Putting $z=0$ into (25), we have

$$
\omega_{0}^{\prime \prime}=\omega=\frac{m c^{2}}{\hbar \sqrt{1-\frac{V^{2}}{c^{2}}}} .
$$

Frequencies of a free particle wave function is a monotone function of its motion velocity, if the physical time continuously. When velocity increases up to its boundary value $C$, frequency increases with no limit infinitely.

Within discrete time situation experiences cardinal changes due to frequency substitution. Dependence of frequency upon particle motion velocity loses its monotone nature.

$$
\omega(V)=\left\{\begin{array}{c}
\omega_{0}^{\prime \prime}(V), \text { if } z=0 \\
\omega_{z}^{\prime}(V), \text { if } z=1,3,5, \ldots \\
\omega_{z}^{\prime \prime},(V), \text { if } z=2,4,6, \ldots
\end{array}\right.
$$

We will define continuous segments of frequency dependence on the velocity as folds. Thus, $\omega_{z}^{\prime \prime}(V)$ areas will be defined as even. We will define continuous segments of 
frequency dependence on the velocity as folds and $\omega_{z}^{\prime}(V)$ areas will be defined as odd ones. Values of critical velocities when frequency substitution takes place within junction points of even folds and odd ones will be found using $\omega_{z}^{\prime}\left(c_{F z}^{\prime \prime}\right)=\omega_{z}^{\prime \prime}\left(c_{F z}^{\prime \prime}\right), \quad \omega_{z}^{\prime}\left(c_{F z}^{\prime}\right)=\omega_{z}^{\prime \prime}\left(c_{F z}^{\prime}\right) \quad$ equalities solving the adequate equations we obtain

$$
\begin{gathered}
\frac{c_{F z}^{\prime \prime}}{c}=\sqrt{1-\frac{\Delta t_{0}}{2 z+1} \frac{m c^{2}}{\pi \hbar}}, \quad z=0,1,2, \ldots \\
\frac{c_{F z}^{\prime}}{c}=\sqrt{1-\frac{\Delta t_{0}}{2 z} \frac{m c^{2}}{\pi \hbar}}, \quad z=1,2,3, \ldots
\end{gathered}
$$

Here $c_{F z}^{\prime \prime}$ means critical velocity within junction point of $z$ even folds with $z+1$ odd folds and $c_{F z}^{\prime}$ means critical velocity within junction point of $z$ odd folds with $z+1$ even folds.

Express ratio $\frac{m c^{2}}{\pi \hbar}$ using Planck time $t_{p l}=\sqrt{\frac{\hbar G}{c^{5}}}$ and Planck mass $m_{p l}=\sqrt{\frac{\hbar c}{G}}$. Multiplying these two values, we obtain $\frac{c^{2}}{\hbar}=\frac{1}{t_{p l} m_{p l}} ;$ hence $\frac{m c^{2}}{\pi \hbar}=\frac{m}{m_{p l}} \frac{\Delta t_{0}}{\pi t_{p l}}$.

Having determined dimensionless constant $\chi$, demonstrating ratio of reduced time quantum $\Delta t_{0} / \pi$ to Planck time $t_{p l}$, we obtain

$$
\begin{gathered}
\chi=\frac{\Delta t_{0}}{\pi t_{p l}}, \\
\frac{c_{F z}^{\prime \prime}}{c}=\sqrt{1-\frac{\chi}{2 z+1} \frac{m}{m_{p l}}}, \quad z=0,1,2, \ldots, \\
\frac{c_{F z}^{\prime}}{c}=\sqrt{1-\frac{\chi}{2 z} \frac{m}{m_{p l}}}, \quad z=1,2,3, \ldots .
\end{gathered}
$$

Putting values of critical velocities into (20) and (21) formulas, determine frequency values within junction points of folds.

$$
\begin{gathered}
\omega\left(c_{F z}^{\prime \prime}\right)=\frac{c^{2}}{\hbar} \sqrt{\frac{m m_{p l}}{\chi(2 z+1)}}, \quad z=0,1,2, \ldots, \\
\omega\left(c_{z F}^{\prime}\right)=0, \quad z=1,2,3, \ldots .
\end{gathered}
$$

Fig. 2 shows graph of particle energy (frequency) dependence upon its motion velocity taking into account time discreteness.

As it is seen time discreteness has cardinal influence on nature of particle energy dependence upon its velocity.

Particle energy (frequency) turns out to be limited. Particle energy with $m$ mass reaches its maximum value within junction point of folds number zero and one, i.e. if $z=0$.

$$
E_{\text {sup }}=c^{2} \sqrt{\frac{m m_{p l}}{\chi}}
$$

Fig. 3 shows dependence of energy upon velocity for particles with different masses.

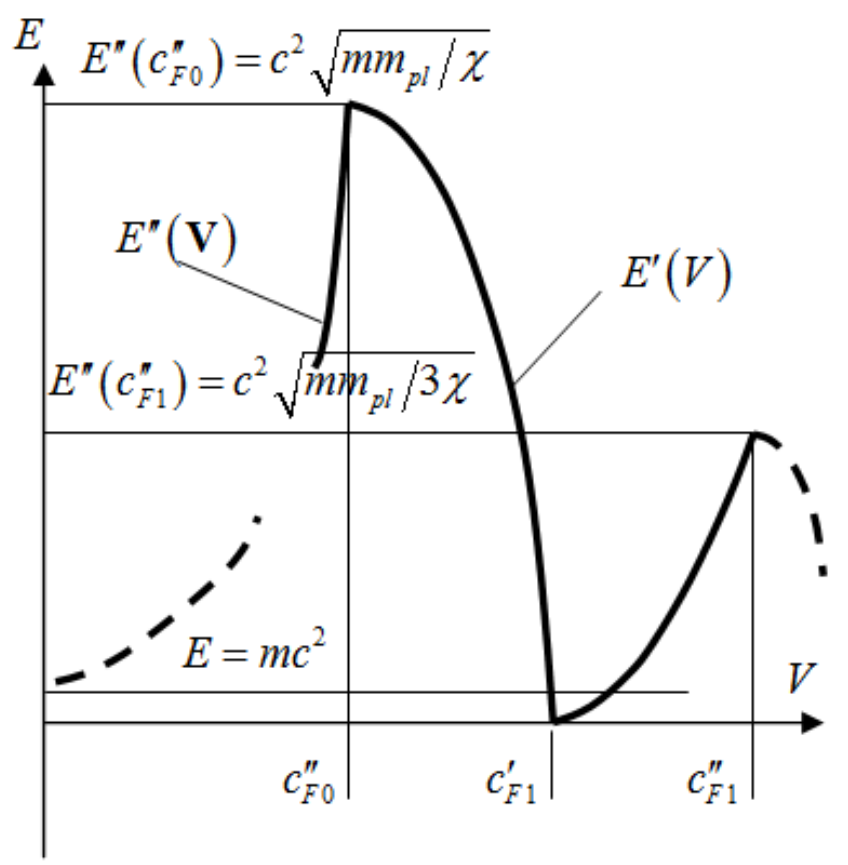

Fig. 2. Anomalous (due to frequency substitution) dependence of particle energy on its velocity.

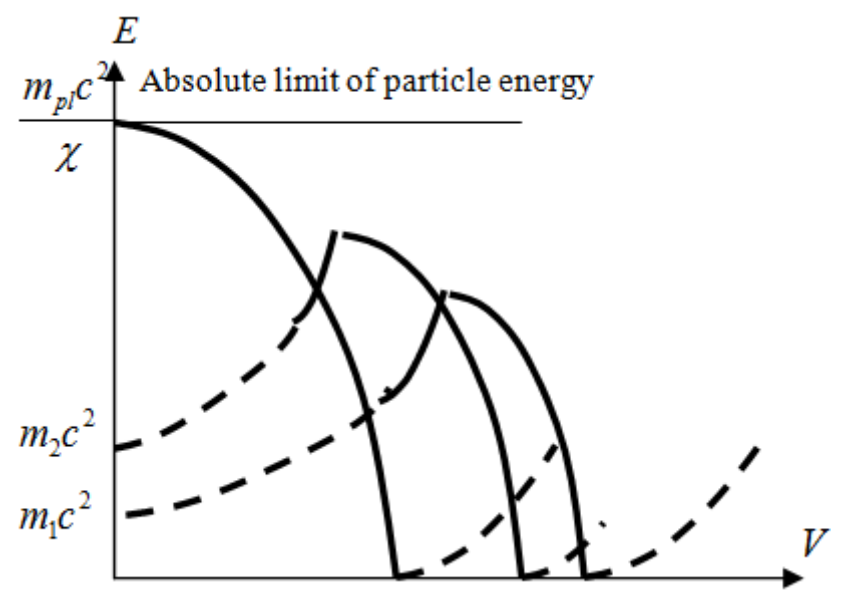

Fig. 3. Dependence of energy upon velocity for particles with different masses.

According to Markov's hypothesis [7] maximum possible mass of a particle at rest is equal to Planck mass. However (33), means that $m_{p l} / \chi$ value is a theoretical boundary of particle mass when frequency substitution takes place, even if its velocity is zero one $\left(c_{F z}^{\prime \prime} / c=0\right)$. Dynamics of such a particle is anomalous within the whole range of its motion velocities.

Zero values of energy (frequency) within junction points for 
odd folds with even ones are second exotic anomaly connected with time discreteness. It means that if particle velocity increases under the effect of external field up to $c_{F 1}^{\prime}$ velocity, it loses its rest energy. If so, its frequency decreases down to zero. If such hyper-ultrarelativistic particles exist, then they lose energy within off folds rather then consume energy while accelerating under the effect of external field.

\section{On Time Quantum Value}

Hypothesis concerning the fact that minimum time interval is numerically equal to the value of Planck time is widely used (but not proved) viewpoint. The above results make it possible to test the hypothesis. If values of time quantum and Planck time are equal, then $\chi=\pi^{-1}$. Using (33) formula, with the help of this $\chi$ value, find maximum energy of known elementary particle, say, proton (938 MeV)

$$
E_{\mathrm{P} \text { sup }}=c^{2} \sqrt{\frac{m_{P} m_{p l}}{\chi}}=c^{2} \sqrt{\pi m_{P} m_{p l}}=5,9959 \cdot 10^{17} \mathrm{eV} .
$$

However, results of observations of space rays of ultrahigh energies show that protons with up to $10^{20} \mathrm{eV}$ energy exist. If we suppose that this is the order of maximum proton energy, then the value of quantum-Planck time ratio is as follows

$$
\chi=\frac{\Delta t_{0}}{\pi t_{p l}}=\frac{m m_{p l} c^{4}}{E_{\text {sup }}^{2}} \simeq 1,144 \cdot 10^{-3} .
$$

It should be noted that dimensionless physical constant reduced constant of fine structure $\alpha / 2 \pi=1,161409732 \cdot 10^{-3}$ is of similar order. Taking into account closeness of two considered dimensionless constants (their difference is not more than two percent) and thinking that our nature follows Occam's rule "not to multiply principles" in the form of two dimensionless constants which difference is less than two percent one can suppose that actually $\chi=\alpha / 2 \pi$, and boundary particle energy with proton mass is $0,985 \cdot 10^{20} \mathrm{eV}$.

In this context the three physical constants - time quantum $\Delta t_{0}$, constant of fine structure $\alpha$, and Planck time $t_{p l}$ - turn out to be connected by the simple ratio

$$
\Delta t_{0}=\frac{\alpha}{2} t_{p l}
$$

Therefore, the maximum frequency of the periodic process in the world is limited to the Nyquist world frequency

$$
F_{w}=\frac{\sigma_{\text {sup }}}{2}=\left(2 \Delta t_{0}\right)^{-1}=\left(\alpha t_{p l}\right)^{-1} .
$$

Accordingly maximum possible mass of a particle at rest is $2 \pi m_{p l} / \alpha$ being approximately three orders more than Planck mass.

\section{Conclusions}

The paper forecasts and obtains mathematical description of physical effect of wave function frequency substitution being direct result of time discreteness. If the effect is found experimentally, then time is discrete. On the contrary, if firm evidence of this effect impossibility or, say, absence of free particle frequency (energy) limitation is found, then time should be considered as continuous.

Modern particle accelerators including LHC cannot still reach energy levels ( $10^{20} \mathrm{eV}$ for protons) required for experimental confirmation of the phenomenon. However, certain paradoxial results of high-energy space rays observations as well as such problems as "hidden mass" and Kozyrev problem [10] about the source of the internal energy of stars can be explained from the viewpoint of time discreteness idea.

1. As it is known, Greisen - Zatsepin - Kuzmin (GZK) paradox is that protons which energy exceeds GZK boundary $\left(5 \cdot 10^{19} \mathrm{eV}\right)$ are available in space rays. Particles with such energy emitted by potentially possible sources due to interaction with background microwave emission should not reach detectors located on the Earth. If we suppose that velocity of hyper-ultrarelativistic particle exceeds critical value $c_{F 0}^{\prime \prime}$ then its energy will decrease (even lower than GZK boundary). Having no contact with background emission, such particle can easily reach ground detectors. Deceleration of such particle in terms of interaction with Earth atmosphere particles will provoke increase in its energy up to boundary value. The fact will be registered by detectors.

2. Problem of hidden mass is seen in the existence of astronomic objects which cannot be observed directly by modern astronomic facilities (those which have not enough electromagnetic or neutrino emissions being satisfactory for intensity observations without their absorption) but those which can be observed indirectly on gravity effects (among other things according to effect of "gravitational lens") upon visible objects.

For the viewpoint of time discreteness hypothesis, reason for the paradox may be as follows. Massive hyper-ultrarelativistic particle moving with a velocity close to $c_{F 1}^{\prime}$ within magnetic field with strength component perpendicular to its motion direction cannot emit much as intensity of synchrotron emission is proportional to a square of particle energy being close to zero in this context. While decelerating being a result of interaction with electromagnetic field or other physical fields such particle consumes energy rather than loses it. Despite the fact that the particle does not disclose itself by electromagnetic wave emission it interacts with the matter surrounding it through gravitation field. It is quite possible that this effect is the essence of hidden mass problem.

3. Particles whose velocity in the gravity field of the star exceeds $c_{F 1}^{\prime}$ give up their rest energy. "Star is a machine that generates energy due to the arrival of its external." [10]. 


\section{References}

[1] Amelino-Camelia G., Relativity in spacetimes with short-distance structure governed by an observer-independent (Planckian) length scale, Int. J. Mod. Phys. D11, 2002, pp. 35-60.

[2] Esposito S., Salesi G., arXiv:1001.0050v1 [physics. hist-ph], 2009.

[3] Guang Ping He, arXiv:0911.2416v2 [quant-ph], 2009.

[4] Shan Gao, arXiv: 1001.5085v2 [physics.gen-ph], 2011.

[5] Abraham Boyarsky, Paweł Góra, Nonobservable space dimensions and the discreteness of time, Chaos, Solitons \& Fractals,V. 24, Issue 1, pp. 13-18, April 2005.
[6] R. Otnes, L. Enochson, Applied time series analysis. Basic techniques. - John Wiley and Sons Inc, 1978, p. 428.

[7] Nyquist H. Certain topics in telegraph transmission theory. AIEE Transaction, p. 617, Apr. 1928.

[8] Shannon C., Communication in the presence of noise, PIRE, $37,1,1949$.

[9] Markov M.A., Progr. Theor. Phys.: Suppl. Commemoration Issue for $30^{\text {th }}$ Anniversary of the Meson Theory by Dr. H. Yukawa, 1965.

[10] Kozyrev N.A. Sources of stellar energy and the theory of the internal structure of stars, Proceedings of the Crimean Astrophysical Observatory, 1948, V.2, 3-43 (in Russian). 